Monitoringverfahren der OECD Working Group on Bribery erfahren möchten; (2) Völkerrechtler und Verwaltungsjuristen, die sich für die rechtsdogmatischen Ausführungen zu internationalen Politikbewertungen interessieren; (3) Politik- und Verwaltungswissenschaftler, die sich über Governance by Information und/oder die Rolle von Verwaltungsinstitutionen im Kontext von Global Governance informieren wollen.

Sebastian Wolf, Konstanz

\title{
Wolfgang Babeck
}

\section{Einführung in das australische Recht mit neuseeländischem Recht.}

Schriftenreihe der Juristischen Schulung, Band 195, Verlag C. H. Beck, München 2011, XXVII, 294 Seiten, Kartoniert, EUR 39,80, ISBN 978-3-406-61959-5

In den letzten 15-20 Jahren ist Australien bei deutschen Studierenden und Referendar(inn)en immer populärer geworden. Beflügelt wurde das unter anderem durch die strenger gewordenen Einreisebedingungen der USA, was eine Hinwendung zu anderen Ländern des Common Law zur Folge hatte, und durch das positive Bild, das die Olympischen Spiele 2000 von Australien zeichneten. Australien bietet Studierenden hervorragende Voraussetzungen, sich Kenntnisse im Recht des Common Law anzueignen und gleichzeitig einen faszinierenden Kontinent zu entdecken. Durch das gesteigerte Interesse am fünften Kontinent sind in den letzten Jahren vermehrt deutsch-australische rechtsvergleichende Themen wissenschaftlich bearbeitet worden. Dr. Wolfgang Babeck, selbst Ausbilder vieler Referendare, möchte diesen und den Studierenden mit Interesse an diesem Erdteil mit seinem Buch einen Leitfaden an die Hand geben.

Das bis auf die beiden australischen Vorworte auf Deutsch geschriebene Werk befasst sich mit einem breiten Themenspektrum aus dem australischen Rechtsystem, nämlich den Grundlagen des australischen Rechts, dem Staats- und Verfassungsrecht, den Grundlagen des Vertragsrechts, dem Immobilienerwerb, dem Mietrecht, den Kreditsicherheiten, dem Schadensersatz- und Deliktsrecht, dem Produkthaftungsrecht, dem Familien- und Erbrecht, dem Handelsrecht, dem Gesellschaftsrecht, dem Recht des Unternehmenskaufs, dem gewerblichen Rechtsschutz, dem Arbeitsrecht, dem australischen Gerichtssystem, dem Zivilverfahren, dem einstweiligen Rechtschutz, dem Insolvenzrecht, dem Verwaltungsrecht, dem Einwanderungsrecht, dem Boden-, Umwelt- und Bauplanungsrecht, dem materiellen Strafrecht und dem Strafprozessrecht. Zusätzlich werden noch das Staats- und Verfassungsrecht, die Grundzüge des Vertragsrechts, des Haftungsrechts, des Gesellschaftsrechts, des Außenwirtschaftsrechts und des gewerblichen Rechtsschutzes Neuseelands dargestellt. Abgerundet wird diese Einführung durch einen Führer zu LL.M.-Studiengängen in Australien und Neuseeland.

Neben der obengenannten Themenfülle beeindruckt das Buch durch die Breite der Zielgruppe (Studierende, Rechtsreferendaren und Wirtschaftsanwälte) sowie durch die namhaften 
Verfasser der Vorworte. Der Autor hat es sich nicht nehmen lassen, die Generalgouverneurin von Australien, Quentin Bryce, sowie den international renommierten ehemaligen High Court-Richter, The Honorable Michael Kirby, um ein Vorwort zu bitten. Bemerkenswert ist ferner die Zahl der Referendare, die an dem Buch mitgewirkt haben (siehe Seite VI und VII). Sie zeugt davon, wie vielen Australienbegeisterten der Verfasser eine Ausbildung auf dem fünften Kontinent ermöglicht hat.

Bei der Breite der Themen vermisst man inhaltlich allerdings eine größere Tiefe. Das Buch bewegt sich in vielen Teilen eher an der Oberfläche und setzt sich mit den eigentlichen rechtsvergleichenden Themen nicht näher auseinander. Wichtige rechtsvergleichende Arbeiten werden zwar in den Literaturübersichten zu den einzelnen Kapiteln aufgeführt, jedoch erfolgt keine inhaltliche Befassung mit diesen, sieht man einmal vom Vorwort Michael Kirbys ab. Die in den Literaturübersichten zitierten Arbeiten befassen sich nicht immer mit den in dem jeweiligen Kapitel behandelten Themen. Auch eine größere Konsistenz zwischen den einzelnen Kapiteln wäre wünschenswert gewesen. Liest man das Buch als Ganzes, so finden sich immer wieder Wiederholungen, die bereits in vorangehenden Kapiteln erläutert wurden. Besser zielführend wäre es daher gewesen, das Buch als Sammlung von Aufsätzen deutschaustralischer Experten unter Herausgeberschaft des Verfassers zu gestalten. Weiterführend wäre ferner ein Verzeichnis der rechtsvergleichenden Arbeiten sowie ein Gesamtliteraturverzeichnis gewesen.

Was das neuseeländische Recht betrifft, so hätte dieses, wie der Autor selbst schreibt (S. 241), ein eigenes Buch verdient. Sicherlich gibt es viele Ähnlichkeiten zwischen dem neuseeländischen und dem australischen Recht. Diese bestehen bspw. aber auch zwischen dem englischen und dem australischen Recht.

Für die Zielgruppe der Studierenden und Referendare ist das Buch jedoch sicher hilfreich, wenn es darum geht, Studien- oder Forschungsschwerpunkte bspw. bei der Fächerwahl vor dem Masterstudium herauszufinden und festzulegen. Für Studierende interessant ist ebenfalls der LL.M.-Studienführer am Ende des Buches, wobei dieser eine eher subjektive Einschätzung hinsichtlich der Qualität der einzelnen Rechtsfakultäten enthält. So kommen einige Fakultäten, bei anders lautenden Rankings, besonders gut im LL.M.-Führer weg. Bei der Erwähnung zweier sogenannter Studienplatzmakler (Agents) auf Seite 268 vermisst man einen Hinweis darauf, dass es sich hierbei um kommerzielle Anbieter handelt und noch weitere diesbezüglich tätig sind. Weiterführend ist hier für Studierende eher die Webseite der australischen Regierung: www.study-in-australia.org/Germany.

Sabine Pittrof, Frankfurt am Main 